\title{
A QUASILINEAR HYPERBOLIC INTEGRODIFFERENTIAL EQUATION RELATED TO A NONLINEAR STRING
}

\author{
BY
}

MELVIN L. HEARD

ABstract. We discuss global existence, boundedness and regularity of solutions to the integrodifferential equation

$$
\begin{gathered}
u_{t t}(t, x)+c(t) u_{t}(t, x)-M\left(\int_{-\infty}^{+\infty}\left|u_{x}(t, s)\right|^{2} d s\right) u_{x x}(t, x)+u(t, x) \\
=h(t, x, u(t, x)), \quad 0 \leq t<\infty, x \in \mathbf{R}, \\
u(0, x)=u_{0}(x), \quad u_{t}(0, x)=u_{1}(x), \quad x \in \mathbf{R} .
\end{gathered}
$$

This type of equation occurs in the study of the nonlinear behavior of elastic strings. We show that if the initial data $u_{0}(x), u_{1}(x)$ is small in a suitable sense, and if the damping coefficient $c(t)$ grows sufficiently fast, then the above equation possesses a globally defined classical solution for forcing terms $h(t, x, u)$ which are sublinear in $u$. In the nonlinearity we require that $M \in C^{1}[0, \infty)$ and, in addition, satisfies $M(\lambda) \geq m_{0}>0$ for all $\lambda \geq 0$.

1. Introduction. We consider the following integrodifferential equation:

$$
\begin{gathered}
u_{t t}(t, x)+c(t) u_{t}(t, x)-M\left(\int_{-\infty}^{+\infty}\left|u_{x}(t, s)\right|^{2} d s\right) u_{x x}(t, x)+u(t, x) \\
=h(t, x, u(t, x)), \quad 0 \leq t<\infty, x \in \mathbf{R}, \\
u(0, x)=u_{0}(x), \quad u_{t}(0, x)=u_{1}(x), \quad x \in \mathbf{R} .
\end{gathered}
$$

Equations of this type have occurred during the study of the nonlinear behavior of elastic strings [14]. A derivation of (1.1) for finite strings is given in [15] where the purely transverse vibration of a linearly elastic stretched string is considered. The basic physical assumptions are that the longitudinal strain of the string is very small and that the tension $F$ is uniform along the string but may vary with time to accomodate changes in arc length of the string. The nonlinearity arises from the assumption that $F$ depends on the arc length $S$ of the string at time $t \geq 0$ by the relation $F=F_{0}+C[(S-L) / L]$ where $F_{0}$ is the minimum tension, $L$ is the minimum length and $C$ is a physical constant.

Our study of (1.1), (1.2) was motivated by a problem posed by J. L. Lions [12] concerning the initial-boundary value problem

$$
\begin{aligned}
& u_{t t}(t, x)-\left(m_{0}+\int_{0}^{\infty}\left|u_{x}(t, s)\right|^{2} d s\right) u_{x x}(t, x)=h(t, x), \\
& \qquad 0 \leq t<\infty, 0 \leq x<\infty,
\end{aligned}
$$

Received by the editors March 3, 1983 and, in revised form, November 9, 1983.

1980 Mathematics Subject Classification. Primary 45K05, 58D25; Secondary 73C50.

Key words and phrases. Integrodifferential equations, evolution equations, stable family of generators, energy estimates, elastic strings. 


$$
\begin{gathered}
u(t, 0)=0, \quad 0 \leq t<\infty, \\
u(0, x)=u_{0}(x), \quad u_{t}(0, x)=u_{1}(x), \quad 0 \leq x<\infty,
\end{gathered}
$$

where $m_{0}>0$ is a constant. By making the appropriate oddness asumptions, we can obtain solutions to (1.3), (1.4), (1.5) from the problem (1.1), (1.2) (cf. Theorem 3). The difficulty with the approach used in $[\mathbf{1 2}]$ is in obtaining the necessary estimates even for the existence of local solutions. In this study we rely on the results of Hughes, Kato and Marsden [8] to obtain local solutions of arbitrary smoothness. This is a relatively straightforward application of $[8]$ and does not require any a priori bounds on $u(t, x)$. We then derive estimates to extend the solution to infinity in the time variable. For this part of the argument we modify some ideas of R. W. Dickey $[4,5]$, who considered a problem similar to (1.3), (1.4), (1.5) but on a bounded interval $0 \leq x \leq L$. Dickey was interested in the infinite system of ordinary differential equations associated with his solution by means of a Fourier sine series. Crucial to his analysis was a certain function $E_{j, N}$ (see $[4$, p. 462]) which acted like a Liapunov function for his system. It is this function that we modify for our purposes.

There are other types of integrodifferential equations similar to (1.1) and we mention, in particular, the one occurring in the study of hinged bars [16]. This type is usually referred to as the extensible beam equation and has been studied before (see, for example, $[\mathbf{2}, \mathbf{3}, \mathbf{6}, \mathbf{7}]$ ). However, in the extensible beam equation the highest order term is linear and fourth order in the space variable. This is a great advantage in the analysis of that equation which is not present in (1.1).

In $\S 2$ we state the abstract result from [8] that is needed for local solutions of (1.1), (1.2). This result is based on the linear theory of evolution equations with time varying generators developed in $[9, \mathbf{1 0}]$. In this linear theory the concept of stable and quasi-stable families of operators plays a central role. To keep our presentation coherent, we recall the definition of a stable family. Let $X$ be a Banach space with norm $\|\cdot\|$ and let $\{\Lambda(t): 0 \leq t \leq T\}$ be a family of closed, linear, densely defined operators in $X$ whose domains $D(\Lambda(t))$ may change with $t$. We say that $\{\Lambda(t): 0 \leq t \leq T\}$ is a stable family on $[0, T]$ if there are constants $M, \beta$ such that

$$
\left\|\left(\Lambda\left(t_{k}\right)+\lambda I\right)^{-1} \cdots\left(\Lambda\left(t_{1}\right)+\lambda I\right)^{-1}\right\| \leq M(\lambda-\beta)^{-k}, \quad \lambda>\beta,
$$

for every finite family $\left\{t_{j}\right\}$ with $0 \leq t_{1} \leq t_{2} \leq \cdots \leq t_{k} \leq T$. This concept will be used in the proof of Theorem 4 .

In $\S 3$ we give a proof of the local existence theorem for (1.1), (1.2). In this theorem no sign condition is made on $c(t)$ and no growth restriction is imposed on $h(t, x, u)$ in the $u$ variable. Our main result is given in $\S 4$, Theorem 4 . We show that if the initial data is sufficiently "small" and if the damping coefficient $c(t)$ grows sufficiently fast, then (1.1), (1.2) will possess globally defined classical solutions for forcing terms $h(t, x, u)$ which are sublinear in $u$. The growth of $c(t)$ depends on $h(t, x, u), M(\lambda)$ as well as the intial data $u_{0}(x), u_{1}(x)$. We can take $c(t)=$ any positive constant, $t \geq 0$, only if $h(t, x, u)=0$. Our arguments differ from the usual type of proofs of this nature in that our small solutions at $t=0$ do not necessarily remain small for all $t>0$. So in $\S 5$ we give some results on the pointwise boundedness of solutions of (1.1), (1.2). The hypotheses stated in that section are sufficiently general enough to hold automatically in the case $h(t, x, u)=0$. 
Finally, we note that our estimates are derived by means of energy methods and therefore can be applied to suitably generalized higher dimensional problems of the form (1.1), (1.2) (cf. [13, Problem 11.10, Chapter 2]). But to keep the exposition clear, we discuss only the one-dimensional problem (1.1), (1.2). We mention also that in the case of $(1.3),(1.4),(1.5)$, where $c(t)=0$, we have no results on the development of singularities of the solution $u(t, x)$. Thus we cannot state for certain that solutions fail to exist for all time $t \geq 0$.

2. Preliminaries. We outline briefly the main conditions from the abstract theory needed to solve (1.1), (1.2). In order to simplify the discussion, we only consider quasilinear evolution equations of the form

$$
d u / d t+A(u) u=f(t, u), \quad 0 \leq t \leq T .
$$

Suppose $X, Y, Z$ are real, separable, reflexive Banach spaces with dense continuous embeddings $Y \rightarrow X \rightarrow Z$. Let $\|\cdot\|_{X},\|\cdot\|_{Y},\|\cdot\|_{Z}$ denote the respective norms on $X, Y, Z$. We let $N(Z)$ be the set of all norms on $Z$ which are equivalent to $\|\cdot\|_{Z}$ and we define a metric on $N(Z)$ by

$$
d\left(\|\cdot\|_{\mu},\|\cdot\|_{\nu}\right)=\log \max \left\{\sup _{z \neq 0} \frac{\|z\|_{\mu}}{\|z\|_{\nu}}, \sup _{z \neq 0} \frac{\|z\|_{\nu}}{\|z\|_{\mu}}\right\} .
$$

Let $W \subset Y$ be a nonempty open set and let $\left\{\|\cdot\|_{(w)}: w \in W\right\}$ be a family of norms in $N(Z)$ which satisfies:

(N) There are positive constants $\lambda_{N}, \mu_{N}$ such that

$$
d\left(\|\cdot\|_{(w)},\|\cdot\|_{Z}\right) \leq \lambda_{N}, \quad d\left(\|\cdot\|_{(w)},\|\cdot\|_{(\tilde{w})}\right) \leq \mu_{N}\|w-\tilde{w}\|_{X}
$$

for all $w, \tilde{w} \in W$.

Let $\{A(w): w \in W\}$ be a family of closed linear operators in $Z$ with dense domains $D(A(w))$. For each $w \in W$, assume that $-A(w)$ generates a $C_{0}$-semigroup $\left\{e^{-e s A(w)}: 0 \leq s<+\infty\right\}$ in $Z$. We assume further that:

(A1) There is a constant $\beta$ such that $\left\|e^{-s A(w)} x\right\|_{(w)} \leq e^{\beta s}\|z\|_{(w)}$ for all $z \in Z$, $w \in W, 0 \leq s<\infty$.

(A2) For each $w \in W$ we have $Y \subset D(A(w))$ and $A(w): Y \rightarrow X$ is a bounded linear operator. Also, there are positive constants $\lambda_{A}, \mu_{A}$ such that $\|A(w)\|_{Y, X} \leq$ $\lambda_{A}$ and $\left\{A(w)-A(\tilde{w})\left\|_{Y, Z} \leq \mu_{A}\right\| w-\tilde{w} \|_{Z}\right.$ for all $w, \tilde{w} \in W$.

(A3) There is a linear isomorphism $S$ of $Y$ onto $Z$ such that for all $w \in W$ we have $S A(w) S^{-1}=A(w)$ on $D(A(w))$.

(A4) There is a dense set $D \subset W$ such that for each $y_{0} \in D$ we have $A(w) y_{0} \in Y$ for all $w \in W$. Also, there is a positive constant $\lambda_{0}$ depending only on $y_{0}$ such that $\left\|A(w) y_{0}\right\|_{Y} \leq \lambda_{0}$ for all $w \in W$.

(f) There is a function $f:[0, T] \times W \rightarrow Y$ and positive constants $\lambda_{f}, \mu_{f}, \mu_{f}^{\prime}$ such that $\|f(t, w)\|_{Y} \leq \lambda_{f}$ and

$$
\|f(t, w)-f(t, \tilde{w})\|_{Z} \leq \mu_{f}\|w-\tilde{w}\|_{Z}, \quad\|f(t, w)-f(t, \tilde{w})\|_{Y} \leq \mu_{f}^{\prime}\|w-\tilde{w}\|_{Y}
$$

for all $0 \leq t \leq T, w$ and $\tilde{w}$ in $W$. Also, the map $t \rightarrow f(t, w)$ is continuous from $[0, T]$ to $\bar{Z}$.

From $[8$, Theorems I, II] we have the following result. 
THEOREM 1. Let (N), (A1)-(A4) and (f) be satisfied. Then, given $u_{0} \in W$ and $t_{0} \in[0, T)$, there is $\delta_{0} \in\left(0, T-t_{0}\right]$ and a unique function $u(t)$, which belongs to $C\left(\left[t_{0}, t_{0}+\delta_{0}\right] ; W\right) \cap C^{1}\left(\left[t_{0}, t_{0}+\delta_{0}\right] ; X\right)$, such that $u\left(t_{0}\right)=u_{0}$ and $u(t)$ satisfies $(2.1)$ for all $t_{0} \leq t \leq t_{0}+\delta_{0}$. We call $u(t)$ a strong solution of (2.1) and if $u_{0}^{n} \rightarrow u_{0}$ in $W$ then the corresponding solutions $u^{n}(t)$ converge to $u(t)$ in the $Y$-norm, uniformly on $\left[t_{0}, t_{0}+\delta_{0}\right]$.

3. Local existence. We first outline our basic assumptions for equation (1.1). Let $s \geq 0$ be an integer and let $H^{s}$ denote the real Sobolev (Hilbert) space of all real-valued measurable functions $\varphi(x)$, defined for $x \in \mathbf{R}$, whose distributional derivatives $D^{k} \varphi$ are square integrable on $\mathbf{R}$ for all $0 \leq k \leq s$. We define a norm on $H^{s}$ by

$$
\|\varphi\|_{s}^{2}=\int_{-\infty}^{+\infty} \sum_{k=0}^{s}\left|D^{k} \varphi(x)\right|^{2} d x .
$$

Let $s \geq 1$ be fixed and let $X, Y, Z$ denote the product (Hilbert) spaces

$$
Z=H^{1} \times H^{0}, \quad X=H^{s} \times H^{s-1}, \quad Y=H^{s+1} \times H^{s}
$$

with norms

$$
\begin{aligned}
\|z\|_{Z}^{2} & =\left\|z^{0}\right\|_{1}^{2}+\left\|z^{1}\right\|_{0}^{2}, \quad z=\left(z^{0}, z^{1}\right) \in Z, \\
\|v\|_{X}^{2} & =\left\|v^{0}\right\|_{s}^{2}+\left\|v^{1}\right\|_{s-1}^{2}, \quad v=\left(v^{0}, v^{1}\right) \in X, \\
\|w\|_{Y}^{2} & =\left\|w^{0}\right\|_{s+1}^{2}+\left\|w^{1}\right\|_{s}^{2}, \quad w=\left(w^{0}, w^{1}\right) \in Y .
\end{aligned}
$$

We make the following assumptions on the functions $M, c$ and $h$ occurring in (1.1):

(M) The function $M \in C^{1}[0, \infty)$ and there is a number $m_{0} \in(0,1)$ such that $M(\lambda) \geq m_{0}>0$ for all $\lambda \geq 0$.

(c) The function $c \in C[0, \infty)$.

(h) The function $h:[0, \infty) \times \mathbf{R} \times \mathbf{R} \rightarrow \mathbf{R}$ is continuous with $s+1$ continuous partial derivatives with respect to $(x, \xi) \in \mathbf{R} \times \mathbf{R}$. Also, for each $T>0$ and each compact interval $K$ there is a constant $C=C(K, T)=0$ such that $\left|D_{(x, \xi)}^{\gamma} h(t, x, \xi)\right| \leq C$ for all $0 \leq t \leq T, x \in \mathbf{R}, \xi \in K, 0 \leq \gamma \leq s+1$. Furthermore, the function $(t, x) \rightarrow h(t, x, 0)$ belongs to $C\left([0, T] ; H^{s}\right)$ for every $T>0$.

We have the following result.

THEOREM 2. Let $s>2$ and $t_{0} \in[0, \infty)$ be given. Let assumptions $(\mathrm{M}),(\mathrm{c})$ and (h) be satisfied. Then for each pair $\left(u_{0}, u_{1}\right) \in H^{s+1} \times H^{s}$ there exists $\delta_{0}>0$ and a unique classical solution $u(t, x)$ which satisfies (1.1) for $t_{0} \leq t \leq t_{0}+\delta_{0}, x \in \mathbf{R}$, with

$$
u\left(t_{0}, x\right)=u_{0}(x), \quad u_{t}\left(t_{0}, x\right)=u_{1}(x), \quad x \in \mathbf{R} .
$$

Proof. For each $w=\left(w^{0}, w^{1}\right) \in Y$ we define

$$
A(w) \varphi=\left(-\varphi^{1},-M\left(\left\|D w^{0}\right\|_{0}^{2}\right) D^{2} \varphi^{0}\right), \quad \varphi \in D(A(w)) \equiv H^{2} \times H^{1} .
$$

Then (1.1), (3.1) can be written as a first order system

$$
\begin{gathered}
d v / d t+A(v(t)) v=f(t, v(t)), \quad t \geq t_{0}, \\
v\left(t_{0}\right)=v_{0}
\end{gathered}
$$


where

and

$$
v=\left(\begin{array}{c}
u \\
u_{t}
\end{array}\right), \quad v_{0}=\left(\begin{array}{c}
u_{0} \\
u_{1}
\end{array}\right)
$$

$$
f(t, w)(x)=\left(\begin{array}{c}
0 \\
h\left(t, x, w^{0}(x)\right)-w^{0}(x)-c(t) w^{1}(x)
\end{array}\right)
$$

for $w=\left(w^{0}, w^{1}\right) \in Y$.

Let $t_{0} \in[0,+\infty)$ be given and choose a fixed constant $T>t_{0}$. Let $R>0$ be given and define $W=\left\{w \in Y:\|w\|_{Y}<R\right\}$. We show that (3.2), (3.3) satisfy the conditions of Theorem 1 . In what follows we let $C_{i}, i=1,2,3, \ldots$, denote generic constants which depend ultimately only on $R$ and $T$. In order to verify hypothesis (N) we define, for each $w=\left(w^{0}, w^{1}\right) \in Y$, norms $\|\cdot\|_{(w)}$ and corresponding inner products $\langle\cdot, \cdot\rangle_{(w)}$ on $Z$ by

$$
\|z\|_{(w)}^{2}=B\left(w ; z^{0}, z^{0}\right)+\left\|z^{1}\right\|_{0}^{2}, \quad\langle v, z\rangle_{(w)}=B\left(w ; v^{0}, z^{0}\right)+\left(v^{1}, z^{1}\right)_{0},
$$

for all $v=\left(v^{0}, v^{1}\right)$ and $z=\left(z^{0}, z^{1}\right)$ in $Z$, where

$$
B(w ; \varphi, \psi)=M\left(\left\|D w^{0}\right\|_{0}^{2}\right) \int_{-\infty}^{+\infty} D \varphi(x) D \psi(x) d x, \quad \varphi, \psi \in H^{1}
$$

By (M) we have

$$
\left|\|z\|_{(w)}^{2}-\|z\|_{(\tilde{w})}^{2}\right| \leq C_{1}\|w-\tilde{w}\|_{Y}\|z\|_{Z}^{2}
$$

for all $z \in Z$ and all $w, \tilde{w} \in W$. On the other hand,

$$
\left|\|z\|_{(w)}^{2}-\|z\|_{(\tilde{w})}^{2}\right| \geq C_{2}\left|\|z\|_{(w)}-\|z\|_{(\tilde{w})}\right|\|z\|_{z} .
$$

Thus we obtain

$$
\left|\|z\|_{(w)}-\|z\|_{(\tilde{w})}\right| \leq C_{3}\|w-\tilde{w}\|_{Y}\|z\|_{Z}
$$

and this implies

$$
d\left(\|\cdot\|_{(w)},\|\cdot\|_{(\tilde{w})}\right) \leq \log \left\{C_{4}\|w-\tilde{w}\|_{Y}+1\right\} \leq C_{5}\|w-\tilde{w}\|_{Y}
$$

for all $w, \tilde{w} \in W$. Also, it is easy to see that $d\left(\|\cdot\|_{(w)},\|\cdot\|_{Z}\right) \leq C_{6}$ for all $w \in W$. This verifies that hypothesis $(\mathrm{N})$ holds.

We prove that (A1) holds with $\beta=0$. First, it is clear that for each $w \in W$ the operator $A(w)$ is closed, linear and densely defined in $Z$. So by the Hille-PhillipsYosida Theorem it suffices to show that for each $w \in W$ we have

$$
\left\|(A(w)+\lambda I)^{-1} z\right\|_{(w)} \leq \lambda^{-1}\|z\|_{(w)}, \quad z \in Z, \lambda>0 .
$$

Integration by parts gives

$$
\langle(A(w)+\lambda I) v, v\rangle_{(w)}=\lambda\|v\|_{(w)}^{2}, \quad v \in D(A(w)), \lambda>0,
$$

so that

$$
\|(A(w)+\lambda I) v\|_{(w)} \geq \lambda\|v\|_{(w)}, \quad v \in D(A(w)), \lambda>0 .
$$

Thus (3.4) will follow from (3.5) once we prove that $A(w)+\lambda I$ maps $H^{2} \times H^{1}$ onto $H^{1} \times H^{0}$. Given $z \in H^{1} \times H^{0}$, the solvability of the system $(A(w)+\lambda I) v=z$ for $v=\left(v^{0}, v^{1}\right) \in H^{2} \times H^{1}$ is equivalent to solving, for each $\varphi \in H^{0}$, the problem

$$
-M\left(\left\|D w^{0}\right\|_{0}^{2}\right) D^{2} v^{0}(x)+\lambda^{2} v^{0}(x)=\varphi(x), \quad-\infty<x<+\infty,
$$




$$
v^{0} \in H^{2} .
$$

If $\varphi$ is $C^{\infty}$ with compact support in $\mathbf{R}$, then we can solve (3.6), (3.7) explicitly by Fourier transforms. The solution $v^{0}$ will also be $C^{\infty}$ and belong to $H^{s}$ for every $s \geq 0$. Thus the operator defined by the left-hand side of (3.6) with domain $H^{2}$ has dense range in $H^{0}$. But by (M) the range of this operator is closed in $H^{0}$. Hence (3.6), (3.7) has the required solution for each $\lambda>0$.

Assumption (A2) is obvious because of the local Lipschitz continuity of $M(\lambda)$. To verify (A3) we define the operator $S$ by

$$
S=\left(\begin{array}{cc}
\left(1-D^{2}\right)^{s / 2} & 0 \\
0 & \left(1-D^{2}\right)^{s / 2}
\end{array}\right) .
$$

We know that $S: H^{s+1} \times H^{s} \rightarrow H^{1} \times H^{0}$ is one-one, onto and continuous, with continuous inverse

$$
S^{-1}=\left(\begin{array}{cc}
\left(1-D^{2}\right)^{-s / 2} & 0 \\
0 & \left(1-D^{2}\right)^{-s / 2}
\end{array}\right) .
$$

We need only show that for each $w \in W$ we have $S A(w)=A(w) S$ on the space $H^{s+2} \times H^{s+1}$. Let $w=\left(w^{0}, w^{1}\right) \in W$ and put $M_{0}=M\left(\left\|D w^{0}\right\|_{0}^{2}\right)$. Then given $v=\left(v^{0}, v^{1}\right) \in H^{s+2} \times H^{s+1}$ we have

$$
(S A(w)-A(w) S) v=\left(0,\left[\left(1-D^{2}\right)^{s / 2},-M_{0} D^{2}\right]\left(v^{0}\right)\right)
$$

where $[$,$] denotes the commutator of two operators. So it suffices to prove that$

$$
\left[\left(1-D^{2}\right)^{s / 2},-M_{0} D^{2}\right](\varphi)=0, \quad \varphi \in H^{s+2} .
$$

This obviously holds for $\varphi \in C_{0}^{\infty}(\mathbf{R})$ and then by closure for $\varphi \in H^{s+2}$. This proves (A3).

Hypothesis (A4) holds with $D=W \cap\left(C_{0}^{\infty}(\mathbf{R}) \times C_{0}^{\infty}(\mathbf{R})\right)$. To verify (f) we first define a function $g:[0, \infty) \times \mathbf{R} \times \mathbf{R} \times \mathbf{R} \rightarrow \mathbf{R}$ by $g(t, x, \xi, \eta)=h(t, x, \xi)-\xi-c(t) \eta$. Then, given $w=\left(w^{0}, w^{1}\right) \in W$, we have, from the definition of $f(t, w)$,

$$
f(t, w)(x)=\left(\begin{array}{c}
0 \\
g\left(t, x, w^{0}(x), w^{1}(x)\right)
\end{array}\right)=\left(\begin{array}{c}
0 \\
g(t, x, w(x))
\end{array}\right) .
$$

So if $0 \leq r \leq s$ we have $f(t, w) \in H^{r+1} \times H^{r}$ if and only if $g(t, \cdot, w(\cdot)) \in H^{r}$ and in this case

$$
\|f(t, w)-f(t, \tilde{w})\|_{H^{r+1} \times H^{r}}=\|g(t, \cdot, w(\cdot))=g(t, \cdot, \tilde{w}(\cdot))\|_{r} .
$$

It follows that the Lipschitz properties of $f(t, w)$ result from corresponding properties for $g(t, \cdot, w(\cdot))$. These properties have been proven in $[\mathbf{8}, \mathbf{1 1}]$ for higher dimensions and so we shall omit the details. We mention, however, the following fact which will be useful later on in $\S 4$. Let $1 \leq r \leq s$ and $B \subset H^{r}$ be a bounded set. Then there is a constant $N>0$ depending on $B$ and $T$ such that

$$
\left\|h_{\xi}(t, \cdot, \varphi(\cdot))\right\|_{r, u l} \leq N\left(1+\|\varphi\|_{r}^{r}\right)
$$

for all $\varphi \in B, 0 \leq t \leq T$. The notation $\|\cdot\|_{r, u l}$ denotes the norm of the uniformly local Sobolev space $H_{u l}^{r}$ introduced in [11]. The proof of (3.8) is accomplished by using the chain rule and following the arguments in $[\mathbf{1 1}, \S 5.2]$. 
We have by (h) that the map $(t, x) \rightarrow g(t, x, 0,0)$ belongs to $C\left([0, T] ; H^{s}\right)$. Thus $f(t, w) \in Y$ with

$$
\|f(t, w)\|_{Y} \leq C_{7}+\sup _{0 \leq t \leq T}\|g(t, \cdot, 0,0)\|_{s}, \quad 0 \leq t \leq T, w \in W .
$$

Given $w=\left(w^{0}, w^{1}\right) \in W$, the map $t \rightarrow f(t, w)$ will be continuous from $[0, T]$ to $Z$ if the map $t \rightarrow h\left(t, \cdot, w^{0}(\cdot)\right)$ belongs to $C\left([0, T] ; H^{0}\right)$. Since $(t, x) \rightarrow h(t, x, 0)$ belongs to $C\left([0, T] ; H^{0}\right)$ we get the desired result from the continuity of $h(t, x, \xi)$ and the Lebesgue Dominated Convergence Theorem. Thus hypothesis (f) is valid.

Using Theorem 1 , it follows that for each $v_{0}=\left(u_{0}, u_{1}\right) \in W$ there is a unique strong solution $v(t)=\left(v^{0}(t), v^{1}(t)\right)$ of $(3.2),(3.3)$ on an interval $\left[t_{0}, t_{0}+\delta_{0}\right]$. Setting $u(t, x)=v^{0}(t)(x)$ gives the required solution of (1.1), (3.1). Q.E.D.

REMARK 1. Let $s \geq 2$ and assume that (M), (c) and (h) are satisfied. Suppose that $\left(u_{0}, u_{1}\right) \in H^{s+1} \times H^{s}$. Then from Theorem 2 it follows that there is a unique classical solution $u(t, x)$ of $(1.1),(1.2)$ defined in a maximal domain $[0, d) \times \mathbf{R}$, $0<d \leq+\infty$. Furthermore, the number $d$ does not depend on $s$. The reason that $d$ is independent of the smoothness $s$ is a result of the fact that the partial derivatives of $u(t, x)$ with respect to $x$ satisfy a linear hyperbolic equation whose coefficients and forcing term are sufficiently smooth in $t$ and $x$.

REMARK 2. Using Theorem 2 we can now state an existence result for the problem (1.3), (1.4), (1.5).

THEOREM 3. Let $s \geq 2$ and consider the problem (1.3), (1.4), (1.5). Let $\tilde{u}_{0}, \tilde{u}_{1}$ denote the odd extensions of $u_{0}, u_{1}$, respectively, to all of $\mathbf{R}$. Define $\tilde{h}(t, x)$ on $[0, \infty) \times \mathbf{R}$ by

$$
\tilde{h}(t, x)= \begin{cases}h(t, x) & \text { if } 0 \leq t<\infty, 0 \leq x<\infty \\ -h(t,-x) & \text { if } 0<t<\infty,-\infty<x<0\end{cases}
$$

Assume that $\left(\tilde{u}_{0}, \tilde{u}_{1}\right) \in H^{s+1} \times H^{s}$ and that $\tilde{h}$ satisfies assumption $(\mathrm{h})$. Then there is a unique classical solution $u(t, x)$ of $(1.3),(1.4),(1.5)$ defined on a maximal domain $[0, d) \times[0, \infty)$.

Proof. We use Theorem 2 to obtain a unique classical solution $v(t, x)$ of the problem

$$
\begin{gathered}
v_{t t}(t, x)-\left(m_{0}+\frac{1}{2} \int_{-\infty}^{+\infty}\left|v_{x}(t, s)\right|^{2} d s\right) v_{x x}(t, x)=\tilde{h}(t, x), \quad 0 \leq t<d, x \in \mathbf{R}, \\
v(0, x)=\tilde{u}_{0}(x), \quad v_{t}(0, x)=\tilde{u}_{1}(x), \quad x \in \mathbf{R} .
\end{gathered}
$$

Setting $u(t, x)=v(t, x)$ for $0 \leq t<d, 0 \leq x<\infty$ gives the desired solution. Q.E.D.

4. Global existence. In this section we show that for certain initial data $\left(u_{0}, u_{1}\right) \in H^{s+1} \times H^{s}$ we can take $d=+\infty$ in Theorem 2 , provided that the function $c(t)$ is sufficiently large. To state our assumptions, we let $0<\varepsilon_{0}<+\infty$ be given and put

$$
c_{0}=\varepsilon_{0} m_{0}^{-1} \sup \left\{\left|M^{\prime}(\lambda)\right|: 0 \leq \lambda \leq \varepsilon_{0} m_{0}^{-1}\right\} .
$$


We strengthen assumption (h) by assuming:

$\left(\mathrm{h}^{*}\right)$ The function $h(t, x, \xi)$ satisfies assumption (h). Furthermore, there exists positive continuous functions $a_{i}(t), i=1,2$, and $h_{j}(t, x), j=0,1$, such that $h_{j} \in$ $C\left([0, T] ; H^{0}\right)$ for each $T>0$ and

$$
|h(t, x, \xi)| \leq a_{1}(t)|\xi|+h_{0}(t, x), \quad\left|h_{x}(t, x, \xi)\right| \leq a_{2}(t)|\xi|+h_{1}(t, x),
$$

for all $0 \leq t<+\infty, x \in \mathbf{R}, \xi \in \mathbf{R}$.

We now define

$$
\begin{gathered}
R(t)=\frac{1}{\sqrt{m_{0}}}\left\{\varepsilon_{0}+\frac{4}{c_{0}} \int_{0}^{t}\left\|h_{0}(\tau)\right\|_{0}^{2} d \tau\right\}^{1 / 2} \exp \left[\frac{2}{c_{0}} \int_{0}^{t} a_{1}^{2}(\tau) d \tau\right], \quad t \geq 0 \\
L(t)=\sup \left\{\left|M^{\prime}(\lambda)\right|: 0 \leq \lambda \leq R^{2}(t)\right\}, \quad t \geq 0 \\
a_{3}(t)=\sup \left\{\left|h_{\xi}(t, x, \xi)\right|: x \in \mathbf{R},|\xi| \leq \sqrt{2} R(t)\right\}, \quad t \geq 0
\end{gathered}
$$

By hypothesis $(\mathrm{h})$ the function $a_{3}(t)$ is finite and lower semicontinuous on $[0,+\infty)$. We further define

$$
\begin{aligned}
K(t)= & \varepsilon_{0}+\frac{2}{m_{0}} \int_{0}^{t}\left[\left(a_{2}(\tau)+a_{3}(\tau)\right) R(\tau)+\left\|h_{1}(\tau)\right\|_{0}\right]^{2} d \tau \\
& +\frac{2}{m_{0}^{2}} \int_{0}^{t}\left[a_{1}(\tau) R(\tau)+\left\|h_{0}(\tau)\right\|_{0}\right] R(\tau) d \tau, \quad t \geq 0
\end{aligned}
$$

We replace assumption (c) by the stronger hypothesis:

$\left(\mathrm{c}^{*}\right)$ The function $c \in C[0, \infty)$ and $c(t)>e^{t} L(t) R(t)\left\{m_{0}^{-1} K(t)\right\}^{1 / 2}$ for all $t \geq 0$. We define

$$
M^{*}(\lambda)=\int_{0}^{\lambda} M(\tau) d \tau, \quad \lambda \geq 0,
$$

and we note that by $(\mathrm{M})$ we have $M^{*}(\lambda) \geq m_{0} \lambda$ for all $\lambda \geq 0$. For each $\varphi \in H^{2}$ and $\psi \in H^{1}$ we let

$$
\Gamma(\varphi, \psi)=m_{0}^{-2}\left\{\|\varphi\|_{2}^{2}+\|\psi\|_{1}^{2}+M^{*}\left(\|D \varphi\|_{0}^{2}\right)\right\} .
$$

To obtain global existence for solutions of $(1.1),(1.2)$ we shall argue by contradiction and assume that $0<d<+\infty$. The following lemma will give the necessary estimates on $u(t, x)$ in order to extend $u(t, \cdot)$ beyond $[0, d)$ thereby contradicting the definition of $d$.

LEMMA 1. Let $s \geq 2$ be fixed and let assumptions $(\mathrm{M}),\left(\mathrm{c}^{*}\right)$ and $\left(\mathrm{h}^{*}\right)$ be satisfied. Let $\left(u_{0}, u_{1}\right) \in H^{s+1} \times H^{s}$ satisfy $\Gamma\left(u_{0}, u_{1}\right) \leq \varepsilon_{0}$ and let $u(t, x)$ be the solution of (1.1), (1.2) on a maximal domain $[0, d) \times \mathbf{R}$. If $0<d<+\infty$, then there exists a finite constant $C_{d}>0$ such that

$$
\left\|D_{x}^{\alpha} u(t)\right\|_{0},\left\|D_{x}^{\beta} u_{t}(t)\right\|_{0},\left\|D_{x}^{\gamma} u_{t t}(t)\right\|_{0} \leq C_{d}, \quad 0 \leq t<d,
$$

for all $0 \leq \alpha \leq s, 0 \leq \beta \leq s-1,0 \leq \gamma \leq s-2$.

ProOF. Theorem 2 guarantees the existence of $u(t, x)$ on $[0, d) \times \mathbf{R}$ which satisfies

$$
u \in C\left([0, d): H^{s+1}\right) \cap C^{1}\left(\left[0, u ; H^{s}\right) \cap C^{2}\left([0, d) ; H^{s-1}\right) .\right.
$$


Thus all derivatives in (4.7) are well defined on $[0, d)$. From (4.5) we have

$$
\begin{aligned}
\frac{d}{d t} M^{*} & \left(\int_{-\infty}^{+\infty}\left|u_{x}(t, \tau)\right|^{2} d \tau\right) \\
& =2 M\left(\int_{-\infty}^{+\infty}\left|u_{x}(t, \tau)\right|^{2} d \tau\right) \int_{-\infty}^{+\infty} u_{x}(t, \tau) u_{x t}(t, \tau) d \tau .
\end{aligned}
$$

We multiply (1.1) by $u_{t}(t, x)$ and integrate $x$ over $\mathbf{R}$. Using (4.1) and (4.8) we obtain

$$
\begin{aligned}
& \frac{1}{2} \frac{d}{d t} \int_{-\infty}^{+\infty} u_{t}^{2} d x+c(t) \int_{-\infty}^{+\infty} u_{t}^{2} d x \\
& \quad+\frac{1}{2} \frac{d}{d t} M^{*}\left(\int_{-\infty}^{+\infty}\left|u_{x}(t, \tau)\right|^{2} d \tau\right)+\frac{1}{2} \frac{d}{d t} \int_{-\infty}^{+\infty} u^{2} d x \\
& \quad \leq \frac{a_{1}^{2}(t)}{\varepsilon} \int_{-\infty}^{+\infty} u^{2} d x+\frac{1}{\varepsilon} \int_{-\infty}^{+\infty}\left|h_{0}(t, x)\right|^{2} d x+2 \varepsilon \int_{-\infty}^{+\infty} u_{t}^{2} d x
\end{aligned}
$$

for every $\varepsilon>0$. Choosing $\varepsilon=\frac{1}{2} c_{0}$ and integrating with respect to $t$ :

$$
\begin{aligned}
&\left\|u_{t}(t)\right\|_{0}^{2}+M^{*}\left(\left\|u_{x}(t)\right\|_{0}^{2}\right)+\|u(t)\|_{0}^{2} \leq\left\|u_{1}\right\|_{0}^{2}+M^{*}\left(\left\|u_{0 x}\right\|_{0}^{2}\right)+\left\|u_{0}\right\|_{0}^{2} \\
&+\frac{4}{c_{0}} \int_{0}^{t} a_{1}^{2}(\tau)\|u(\tau)\|_{0}^{2} d \tau+\frac{4}{c_{0}} \int_{0}^{t}\left\|h_{0}(\tau)\right\|_{0}^{2} d \tau, \quad 0 \leq t<d .
\end{aligned}
$$

It follows from (4.2) that

$$
\|u(t)\|_{0},\left\|u_{t}(t)\right\|_{0},\left\|u_{x}(t)\right\|_{0} \leq R(t), \quad 0 \leq t<d .
$$

We let $H(t, x)=h(t, x, u(t, x))$; then by $(4.1)$

$$
|H(t, x)| \leq a_{1}(t)|u(t, x)|+h_{0}(t, x), \quad(t, x) \in[0, d) \times \mathbf{R} .
$$

So for each $t \in[0, d)$ the map $x \rightarrow H(t, x)$ belongs to $L^{2}$. Thus the FourierPlancherel transform $\hat{H}(t, \xi)$ of $H(t, x)$ with respect to $x$ is well defined. We also let

$$
\begin{gathered}
M_{0}(t)=M\left(\int_{-\infty}^{+\infty}\left|u_{x}(t, \tau)\right|^{2} d \tau\right), \quad 0 \leq t<d, \\
E(t, \xi)=\frac{\left|\hat{u}_{t}(t, \xi)\right|^{2}}{\xi^{2} M_{0}(t)+1}+|\hat{u}(t, \xi)|^{2}, \quad 0 \leq t<d, \xi \in \mathbf{R} .
\end{gathered}
$$

From equation (1.1) we have

$$
\frac{\partial}{\partial t} E(t, \xi)=\frac{-2 c(t)\left|\hat{u}_{t}\right|^{2}}{\xi^{2} M_{0}(t)+1}-\frac{\xi^{2} M_{0}^{\prime}(t)\left|\hat{u}_{t}\right|^{2}}{\left(\xi^{2} M_{0}(t)+1\right)^{2}}+\frac{2 \operatorname{Re}\left\{\hat{H} \hat{\hat{u}}_{t}\right\}}{\xi^{2} M_{0}(t)+1}
$$

so that

$$
\frac{\partial E}{\partial t}(t, \xi) \leq-\left\{2 c(t)-\frac{\left|M_{0}^{\prime}(t)\right|}{M_{0}(t)}\right\} \frac{\left|\hat{u}_{t}\right|^{2}}{\xi^{2} M_{0}(t)+1}+\frac{2 \operatorname{Re}\left\{\hat{H} \overline{\hat{u}}_{t}\right\}}{\xi^{2} M_{0}(t)+1}
$$

for all $0 \leq t<d, \xi \in \mathbf{R}$. It follows that

$$
\frac{\partial E}{\partial t}(t, \xi) \leq \frac{2 \operatorname{Re}\left\{\hat{H} \overline{\hat{u}}_{t}\right\}}{\xi^{2} M_{0}(t)+1}, \quad \xi \in \mathbf{R},
$$


for all $t$ satisfying

$$
2 c(t)-\left|M_{0}^{\prime}(t)\right| / M_{0}(t) \geq 0 .
$$

From (4.10) and assumption $\left(\mathrm{c}^{*}\right)$ we have

$$
\frac{1}{2} \frac{\left|M_{0}^{\prime}(0)\right|}{M_{0}(0)} \leq \varepsilon_{0} m_{0}^{-1}\left|M^{\prime}\left(\left\|u_{0 x}\right\|_{0}^{2}\right)\right|<c(0)
$$

so that the number

$$
t^{*}=\sup \left\{t \in[0, d): 2 c(\tau)-\left|M_{0}(\tau)\right| / M_{0}(\tau)>0,0 \leq \tau \leq t\right\}
$$

is well defined. We claim that $t^{*}=d$. Suppose this is false, then $0<t^{*}<d$ and

$$
2 c\left(t^{*}\right)-\left|M_{0}^{\prime}\left(t^{*}\right)\right| / M_{0}\left(t^{*}\right)=0 .
$$

It follows from (4.11), (4.12) that

$$
\left|\hat{u}_{t}(t, \xi)\right|^{2} \leq\left(\xi^{2} M_{0}(t)+1\right)\left[E(0, \xi)+2 \int_{0}^{t} \frac{\operatorname{Re}\left\{\hat{H}(\tau, \xi) \overline{\hat{u}}_{t}(\tau, \xi)\right\}}{\xi^{2} M_{0}(\tau)+1} d \tau\right]
$$

for all $0 \leq t \leq t^{*}, \xi \in \mathbf{R}$. We multiply (4.16) by $\xi^{2}$ and integrate the result over $\mathbf{R}$ to obtain

$$
\begin{aligned}
&\left\|u_{x t}(t)\right\|_{0}^{2} \leq M_{0}(t)\left[\int_{-\infty}^{+\infty} \xi^{4} E(0, \xi) d \xi+2 \int_{-\infty}^{+\infty} \xi^{4} \int_{0}^{t} \frac{\operatorname{Re}\left\{\hat{H}(\tau, \xi) \overline{\hat{u}}_{t}(\tau, \xi)\right\}}{\xi^{2} M_{0}(\tau)+1} d \tau d \xi\right] \\
&+\int_{-\infty}^{+\infty} \xi^{2} E(0, \xi) d \xi+2 \int_{-\infty}^{+\infty} \xi^{2} \int_{0}^{t} \frac{\operatorname{Re}\left\{\hat{H}(\tau, \xi) \overline{\hat{u}}_{t}(\tau, \xi)\right\}}{\xi^{2} M_{0}(\tau)+1} d \tau d \xi, \\
& 0 \leq t \leq t^{*} .
\end{aligned}
$$

From (4.11) we have

$$
\begin{gathered}
\int_{-\infty}^{+\infty} \xi^{2} E(0, \xi) d \xi \leq m_{0}^{-1}\left\|u_{1}\right\|_{0}^{2}+\left\|u_{0 x}\right\|_{0}^{2} \\
\int_{-\infty}^{+\infty} \xi^{4} E(0, \xi) d \xi \leq m_{0}^{-1}\left\|u_{1 x}\right\|_{0}^{2}+\left\|u_{0 x x}\right\|_{0}^{2} .
\end{gathered}
$$

Also by Fubini's theorem,

$$
\begin{gathered}
\int_{-\infty}^{+\infty} \xi^{2} \int_{0}^{t} \frac{\operatorname{Re}\left\{\hat{H}(\tau, \xi) \overline{\hat{u}}_{t}(\tau, \xi)\right\}}{\xi^{2} M_{0}(\tau)+1} d \tau d \xi \leq \frac{1}{m_{0}} \int_{0}^{t}\|H(\tau)\|_{0}\left\|u_{t}(\tau)\right\|_{0} d \tau, \\
\int_{-\infty}^{+\infty} \xi^{4} \int_{0}^{t} \frac{\mid \operatorname{Re}\left\{\hat{H}(\tau, \xi) \overline{\hat{u}}_{t}(\tau, \xi)\right\}}{\xi^{2} M_{0}(\tau)+1} d \tau d \xi \leq \frac{1}{m_{0}} \int_{0}^{t}\left\|H_{x}(\tau)\right\|_{0}^{2} d \tau+\int_{0}^{t} \frac{\left\|u_{x t}(\tau)_{0}^{2}\right\|}{M_{0}(\tau)} d \tau
\end{gathered}
$$

Therefore,

$$
\begin{aligned}
\frac{\left\|u_{x t}(t)\right\|_{0}^{2}}{M_{0}(t)} \leq & m_{0}^{-1}\left\|u_{1 x}\right\|_{0}^{2}+\left\|u_{0 x x}\right\|_{0}^{2}+m_{0}^{-1}\left(m_{0}^{-1}\left\|u_{1}\right\|_{0}^{2}+\left\|u_{0 x}\right\|_{0}^{2}\right) \\
& +\frac{2}{m_{0}} \int_{0}^{t}\left\|H_{x}(\tau)\right\|_{0}^{2} d \tau+\frac{2}{m_{0}^{2}} \int_{0}^{t}\|H(\tau)\|_{0}\left\|u_{t}(\tau)\right\|_{0} d \tau \\
& +2 \int_{0}^{t} \frac{\left\|u_{x t}(\tau)\right\|_{0}^{2}}{M_{0}(\tau)} d \tau
\end{aligned}
$$


for $0 \leq t \leq t^{*}$. Now by $(4.1),(4.9)$

$$
\int_{0}^{t}\|H(\tau)\|_{0}\left\|u_{t}(\tau)\right\|_{0} d \tau \leq \int_{0}^{t}\left(a_{1}(\tau) R(\tau)+\left\|h_{0}(\tau)\right\|_{0}\right) R(\tau) d \tau .
$$

Also by (4.9) and the Sobolev Embedding Theorem [1, Lemma 5.15], we have

$$
|u(t, x)| \leq \sqrt{2} R(t), \quad 0 \leq t<d, x \in \mathbf{R} .
$$

Hence by (4.3)

$$
\left|\frac{\partial h}{\partial \xi}(t, x, u(t, x))\right| \leq a_{3}(t), \quad 0 \leq t<d, x \in \mathbf{R},
$$

and from (4.1), (4.9), (4.19) we obtain

$$
\int_{0}^{t}\left\|H_{x}(\tau)\right\|_{0}^{2} d \tau \leq \int_{0}^{t}\left[\left(a_{2}(\tau)+a_{3}(\tau)\right) R(\tau)+\left\|h_{1}(\tau)\right\|_{0}\right]^{2} d \tau
$$

Since

$$
m_{0}^{-1}\left\|u_{1 x}\right\|_{0}^{2}+\left\|u_{0 x x}\right\|_{0}^{2}+\dot{m}_{0}^{-1}\left(m_{0}^{-1}\left\|u_{1}\right\|_{0}^{2}+\left\|u_{0 x}\right\|_{0}^{2}\right) \leq \Gamma\left(u_{0}, u_{1}\right) \leq \varepsilon_{0},
$$

it follows from (4.4) and (4.17) that

$$
\frac{\left\|u_{x t}(t)\right\|_{0}^{2}}{M_{0}(t)} \leq K(t)+2 \int_{0}^{t} \frac{\left\|u_{x t}(\tau)\right\|_{0}^{2}}{M_{0}(\tau)} d \tau, \quad 0 \leq t \leq t^{*} .
$$

By Gronwall's inequality we obtain

$$
\left\|u_{x t}(t)\right\|_{0}^{2} \leq M_{0}(t) K(t) e^{2 t}, \quad 0 \leq t \leq t^{*} .
$$

From (4.10), (4.20) and $\left(\mathrm{c}^{*}\right)$ we then have

$$
\frac{\left|M_{0}^{\prime}(t)\right|}{M_{0}(t)} \leq \frac{2 L(t) R(t)}{M_{0}(t)}\left\|u_{x t}(t)\right\|_{0}<2 c(t), \quad 0 \leq t \leq t^{*} .
$$

This contradicts (4.13) so that we must have $t^{*}=d$. Thus we obtain a constant $C_{d}>0$ such that

$$
\|u(t)\|_{0},\left\|u_{t}(t)\right\|_{0},\left\|u_{x}(t)\right\|_{0},\left\|u_{x t}(t)\right\|_{0} \leq C_{d}, \quad 0 \leq t<d .
$$

To estimate the higher derivatives of $u(t, x)$, we put $u^{\alpha}(t, x)=D_{x}^{\alpha} u(t, x)$ wher $\epsilon$ $1 \leq \alpha \leq s-1$. Then we know that

$$
u^{\alpha} \in C\left([0, d) ; H^{2}\right) \cap C^{1}\left([0, d) ; H^{1}\right) \cap C^{2}\left([0, d) ; H^{0}\right)
$$

and from (1.1)

$$
\begin{gathered}
u_{t t}^{\alpha}(t, x)+c(t) u_{t}^{\alpha}(t, x)-M_{0}(t) u_{x x}^{2}(t, x)+u^{\alpha}(t, x) \\
=D_{x}^{\alpha} h(t, x, u(t, x)), \quad 0 \leq t<d, x \in \mathbf{R}, \\
u^{\alpha}(0, x)=D_{x}^{\alpha} u_{0}(x), \quad u_{t}^{\alpha}(0, x)=D_{x}^{\alpha} u_{1}(x), \quad x \in \mathbf{R} .
\end{gathered}
$$

Because of (4.21) we can carry out standard energy estimates on (4.22) to obtain the necessary $L^{2}$ bounds. We illustrate for the case $\alpha=1$, the other cases being 
handled exactly in the same manner. First, we multiply (4.22) by $u_{x t}$ and integrate $x$ over R. Using (4.1), (4.3) we obtain

$$
\begin{aligned}
\frac{1}{2} \frac{d}{d t} \int_{-\infty}^{+\infty} u_{x t}^{2} d x+c(t) \int_{-\infty}^{+\infty} u_{x t}^{2} d x \\
\quad+\frac{1}{2} \frac{d}{d t}\left\{M_{0}(t) \int_{-\infty}^{+\infty} u_{x x}^{2} d x\right\}+\frac{1}{2} \frac{d}{d t} \int_{-\infty}^{+\infty} u_{x}^{2} d x \\
\leq \leq \frac{1}{2} M_{0}^{\prime}(t) \int_{-\infty}^{+\infty} u_{x x}^{2} d x+\frac{a_{2}^{2}(t)}{\varepsilon} \int_{-\infty}^{+\infty} u^{2} d x+\frac{a_{3}^{2}(t)}{\varepsilon} \int_{-\infty}^{+\infty} u_{x}^{2} d x \\
\quad+\frac{1}{\varepsilon} \int_{-\infty}^{+\infty}\left|h_{1}(t, x)\right|^{2} d x+3 \varepsilon \int_{-\infty}^{+\infty} u_{x t}^{2} d x,
\end{aligned}
$$

for every $\varepsilon>0$. By (4.10), (4.20) the constant

$$
M_{d}^{\prime}=\sup \left\{\left|M_{0}^{\prime}(t)\right|: 0 \leq t<d\right\}
$$

is finite. So choosing $\varepsilon=c_{0} / 3$ we obtain

$$
\begin{aligned}
\left\|u_{x t}(t)\right\|_{0}^{2}+m_{0}\left\|u_{x x}(t)\right\|_{0}^{2}+\left\|u_{x}(t)\right\|_{0}^{2} \leq & \left\|u_{1 x}\right\|_{0}^{2}+\left\|u_{0 x}\right\|_{0}^{2}+M\left(\left\|u_{0 x}\right\|_{0}^{2}\left\|u_{0 x x}\right\|\right)_{0}^{2} \\
& +\frac{6 C_{d}^{2}}{c_{0}} \int_{0}^{t}\left[a_{2}^{2}(\tau)+a_{3}^{2}(\tau)+\left\|h_{1}(\tau)\right\|_{0}^{2}\right] d \tau \\
& +M_{d}^{\prime} \int_{0}^{t}\left\|u_{x x}(\tau)\right\|_{0}^{2} d \tau, \quad 0 \leq t<d .
\end{aligned}
$$

It follows from Gronwall's inequality that

$$
\left\|u_{x x}(t)\right\|_{0} \leq C_{d}, \quad 0 \leq t<d,
$$

for an appropriate constant $C_{d}>0$. Using the equation (1.1) we then obtain an estimate on $u_{t t}(t, x)$,

$$
\left\|u_{t t}(t)\right\|_{0} \leq C_{d}, \quad 0 \leq t<d
$$

This proves the lemma in the case $s=2$. The other cases $s>2$ proceed in a similar manner. We multiply $(4.22)$ by $u_{t}^{\alpha}(t, x)$ and integrate:

$$
\begin{aligned}
\frac{1}{2} \frac{d}{d t} \int_{-\infty}^{+\infty}\left(u_{t}^{\alpha}(t, x)\right)^{2} d x+c(t) \int_{-\infty}^{+\infty}\left(u_{t}^{\alpha}(t, x)\right)^{2} d x \\
\quad+\frac{1}{2} \frac{d}{d t}\left\{M_{0}(t) \int_{-\infty}^{+\infty}\left(u_{x}^{\alpha}(t, x)\right)^{2} d x\right\}+\frac{1}{2} \frac{d}{d t} \int_{-\infty}^{+\infty}\left(u^{\alpha}(t, x)\right)^{2} d x \\
=\frac{1}{2} M_{0}^{\prime}(t) \int_{-\infty}^{+\infty}\left(u_{x}^{\alpha}(t, x)\right)^{2} d x+\int_{-\infty}^{+\infty} D_{x}^{\alpha} h(t, x, u(t, x)) u_{t}^{\alpha}(t, x) d x
\end{aligned}
$$

By the chain rule

$$
D_{x}^{\alpha} h(t, x, u(t, x))=\frac{\partial^{\alpha} h}{\partial x^{\alpha}}(t, x, u(t, x))+N
$$

where $N$ is the sum of products of $(x, \xi)$-derivatives of $h$ evaluated at $(t, x, u(t, x))$ times $x$-derivatives of $u$. Thus the integral $\int_{-\infty}^{+\infty} N \cdot u_{t}^{\alpha}(t, x) d x$ can easily be estimated by using the Cauchy-Schwarz inequality on products of derivatives of $u$ and 
the $L^{\infty}$-norm on derivatives of $h$. To use the Cauchy-Schwarz inequality on the integral

$$
\int_{-\infty}^{+\infty} \frac{\partial^{\alpha} h}{\partial x^{\alpha}}(t, x, u(t, x)) u_{t}^{\alpha}(t, x) d x
$$

we need an $L^{2}$-estimate on $\partial^{\alpha} h(t, x, u(t, x)) / \partial x^{\alpha}$. Put $h^{\alpha}=\partial^{\alpha} h / \partial x^{\alpha}$, then

$$
h^{\alpha}(t, x, u(t, x))=h^{\alpha}(t, x, 0)+\left(\int_{0}^{1} \frac{\partial h^{\alpha}}{\partial \xi}(t, x, \lambda u(t, x)) d \lambda\right) u(t, x) .
$$

Therefore

$$
\left\|h^{\alpha}(t, \cdot, u(t, \cdot))\right\|_{0} \leq\left\|h^{\alpha}(t, \cdot, 0)\right\|_{0}+h_{d}^{\alpha}\|u(t)\|
$$

where the constant $h_{d}^{\alpha}$ is defined by

$$
h_{d}^{\alpha}=\sup \left\{\left|\frac{\partial h^{\alpha}}{\partial \xi}(t, x, \xi)\right|: 0 \leq t \leq d, x \in \mathbf{R},|\xi| \leq \sqrt{2} R(d)\right\} .
$$

By assumption (h) we have $h_{d}^{\alpha}<\infty$. This proves the lemma. Q.E.D.

THEOREM 4. Let $s \geq 3$ be fixed (see Remark 3 ) and let assumptions (M), ( $\left.\mathrm{c}^{*}\right)$ and $\left(\mathrm{h}^{*}\right)$ be satisfied. Let $\left(u_{0}, u_{1}\right) \in H^{s+1} \times H^{s}$ satisfy $\Gamma\left(u_{0}, u_{1}\right) \leq \varepsilon_{0}$, where $\Gamma$ is defined by (4.6) and $\varepsilon_{0}>0$ is given in (4.2), (4.4). Then there is a unique classical solution $u(t, x)$ of $(1.1),(1.2)$ on $[0, \infty) \times \mathbf{R}$ which satisfies

$$
u \in C\left([0, \infty) ; H^{s+1}\right) \cap C^{-1}\left([0, \infty) ; H^{s}\right) \cap C^{2}\left([0, \infty) ; H^{s-1}\right) .
$$

Proof. We argue by contradiction and suppose that $u(t, x)$ is defined on a maximal domain $[0, d) \times \mathbf{R}$, where $0<d<+\infty$. Let $M_{0}(t)$ be defined by $(4.10)$ and define the operator $\Lambda(t)$ by

$$
\begin{gathered}
\Lambda(t)=\left(\begin{array}{cc}
0 & -I \\
-M_{0}(t) D_{x}^{2} & 0
\end{array}\right), \\
D(\Lambda(t))=H^{2} \times H^{1}, \quad 0 \leq t<d .
\end{gathered}
$$

Let $Y_{0}=H^{3} \times H^{2}, X_{0}=H^{2} \times H^{1}, Z=H^{1} \times H^{0}$ and define $f$ on $[0, \infty) \times Y_{0}$ by

$$
f(t, w)(x)=\left(\begin{array}{c}
0 \\
h\left(t, x, w^{0}(x)\right)-w^{0}(x)-c(t) w^{1}(x)
\end{array}\right)
$$

for $w=\left(w^{0}, w^{1}\right) \in Y_{0}$. Let $v(t)=\left(u(t, \cdot), u_{t}(t, \cdot)\right)$ and $v_{0}=\left(u_{0}, u_{1}\right)$. Then $v(t)$ is the unique strong solution in $Z$ of the Cauchy problem

$$
\begin{gathered}
d v / d t+\Lambda(t) v=f(t, v(t)), \quad 0 \leq t<d, \\
v(0)=v_{0}
\end{gathered}
$$

Let $C_{d}$ denote the constant given in (4.7), let $M_{0}^{\prime}$ be the constant defined by (4.23) and define $M_{d}=\sup \left\{M(\lambda): 0 \leq \lambda \leq C_{d}^{2}\right\}$. Now $\{\Lambda(t): 0 \leq t<d\}$ is a family of closed, linear, densely defined operators in $Z$. Also, using the arguments of $\S 3$, it is easy to see that $\{\Lambda(t): 0 \leq t<d\}$ is a stable family in $Z$ on each compact subinterval $[a, b] \subset[0, d)$ with stability index $\{M, 0\}$, where

$$
M=\exp 2\left[\lambda_{N}+\mu_{N}(b-a)\right]
$$


(see [8, Lemma 2.1]). Here the constants $\lambda_{N}, \mu_{N}$ are defined by

$$
\lambda_{N}=\max \left\{m_{0}^{-1 / 2},\left(M_{d}+1\right)^{1 / 2}\right\}, \quad \mu_{N}=\frac{1}{2} m_{0}^{-1} M_{d}^{\prime} .
$$

We remark that the family of norms $\left\{\|\cdot\|_{(t)}: 0 \leq t<d\right\}$ associated with $\{\Lambda(t): 0 \leq$ $t<d\}$ is given by

$$
\|z\|_{(t)}^{2}=M_{0}(t) \int_{-\infty}^{+\infty}\left|D z^{0}(x)\right|^{2} d x+\int_{-\infty}^{+\infty}\left|z^{1}(x)\right|^{2} d x, \quad z=\left(z^{0}, z^{1}\right) \in Z .
$$

We let $S: Y_{0} \rightarrow Z$ be the linear isomorphism given by

$$
S=\left(\begin{array}{cc}
1-D_{x}^{2} & 0 \\
0 & 1-D_{x}^{2}
\end{array}\right)
$$

The arguments of $\S 3$ show that

$$
S \Lambda(t) S^{-1}=\Lambda(t) \quad \text { on } D(\Lambda(t))=H^{2} \times H^{1} .
$$

Since the mapping $t \rightarrow \Lambda(t)$ is norm continuous from each compact subinterval $[a, b] \subset[0, d)$ to $\mathcal{L}\left(Y_{0}, Z\right)$, it follows from [10] that there is a unique evolution operator $\{U(t, \tau): 0 \leq \tau \leq t<d\}$ associated with $\{\Lambda(t): 0 \leq t<d\}$ such that the solution $v(t)$ of $(4.26),(4.27)$ satisfies

$$
v(t)=U(t, 0) v_{0}+\int_{0}^{t} U(t, \tau) f(\tau, v(\tau)) d \tau, \quad 0 \leq t<d .
$$

The operator $U(t, \tau)$ leaves $Y_{0}$ invariant, it is strongly continuous on $Y_{0}$ and satisfies the estimate (see [8, Lemma 2.6])

$$
\|U(t, \tau)\|_{Z} \leq \exp 2\left[\lambda_{N}+\mu_{N}(t-\tau)\right], \quad 0 \leq \tau \leq t<d .
$$

To estimate $U(t, \tau)$ on $Y_{0}$ we note that (4.28) and [10, Lemma 2] imply that

$$
U(t, \tau)=S U(t, \tau) S^{-1} \quad \text { on } Z, \quad 0 \leq \tau \leq t<d .
$$

Hence

$$
\|U(t, \tau)\|_{Y_{0}} \leq \lambda_{S} \lambda_{S}^{\prime} \exp 2\left[\lambda_{N}+\mu_{N}(t-\tau)\right], \quad 0 \leq \tau \leq t<d,
$$

where $\lambda_{S}=\|S\|, \lambda_{S}^{\prime}=\left\|S^{-1}\right\|$.

We shall now prove that

$$
\lim _{t \rightarrow d^{-}} v(t)=v_{d}
$$

exists in the space $Y_{0}$ and

$$
\lim _{t \rightarrow d^{-}} v^{\prime}(t)=v_{d}^{\prime}
$$

exists in the space $X_{0}$. By the local existence result Theorem 2, we can then solve the Cauchy problem

$$
\begin{aligned}
d v / d t+A(v(t)) v & =f(t, v(t)), \quad t \geq d, \\
v(d) & =v_{d},
\end{aligned}
$$

in some interval $\left[d, d^{\prime}\right]$, where $d^{\prime}>d$, in the space $Y_{0}$. Thus we will have a continuation $\tilde{u}(t, x)$ of $u(t, x)$ to a larger domain $\left[0, d^{\prime}\right] \times \mathbf{R}$ satisfying the smoothness condition

$$
\tilde{u} \in C\left(\left[0, d^{\prime}\right] ; H^{3}\right) \cap C^{1}\left(\left[0, d^{\prime}\right] ; H^{2}\right) \cap C\left(\left[0, d^{\prime}\right] ; H^{1}\right)
$$


since we only have $v_{d} \in Y_{0}=H^{3} \times H^{2}$. However, $\tilde{u}(0, x)=u_{0}(x)$ and $\tilde{u}_{t}(0, x)=$ $u_{1}(x)$ with $\left(u_{0}, u_{1}\right) \in H^{s+1} \times H^{s}$ and by Remark 1 the maximal domain is independent of $s$. Hence $\tilde{u}$ satisfies the same smoothness condition as $u$,

$$
\tilde{u} \in C\left(\left[0, d^{\prime}\right] ; H^{s+1}\right) \cap C^{1}\left(\left[0, d^{\prime}\right] ; H^{s}\right) \cap C^{2}\left(\left[0, d^{\prime}\right] ; H^{s-1}\right) .
$$

This contradicts the definition of $d$.

Let $0 \leq t \leq t+h<d$. Then by (4.29)

$$
\begin{aligned}
v(t+h)-v(t)= & {[U(t+h, 0)-U(t, 0)] v_{0}+\int_{t}^{t+h} U(t+h, \tau) f(\tau, v(\tau)) d \tau } \\
& +\int_{0}^{t}[U(t+h, \tau)-U(t, \tau)] f(\tau, v(\tau)) d \tau
\end{aligned}
$$

We first show that

$$
\lim _{t, t+h \rightarrow d}\left\|[U(t+h, 0)-U(t, 0)] v_{0}\right\|_{Y_{0}}=0 .
$$

In the estimations to be made below, we let $C_{i}, i \geq 1$, denote generic constants which depend only on $d$. Since $\|S w\|_{Z}$ is equivalent to $\|w\|_{Y_{0}}$ for $w \in Y_{0}$, it follows from (4.31) that

$$
\left\|[U(t+h, 0)-U(t, 0)] v_{0}\right\|_{Y_{0}} \leq C_{1}\left\|[U(t+h, 0)-U(t, 0)] S v_{0}\right\|_{Z} .
$$

Hence it suffices to prove that

$$
\lim _{t, t+h \rightarrow d}\|[U(t+h, 0)-U(t, 0)] z\|_{Z}=0, \quad z \in Z .
$$

Given $y \in Y_{0}$, we have

$$
U(t, \tau) y=y-\int_{\tau}^{t} \Lambda(\sigma) U(\sigma, \tau) y d \sigma, \quad 0 \leq \tau \leq t<d .
$$

Since

$$
\|\Lambda(t)\|_{Y_{0}, Z}=\sup _{\|y\|_{Y_{0}} \leq 1}\|\Lambda(t) y\|_{Z} \leq\left(1+M_{d}^{2}\right)^{1 / 2}, \quad 0 \leq t<d,
$$

it follows from (4.37) that

$$
\lim _{t, t+h \rightarrow d}\|[U(t+h, 0)-U(t, 0)] y\|_{Z}=0, \quad y \in Y_{0} .
$$

Since $Y_{0}$ is dense in $Z$ and since (4.30) holds, it follows that (4.36) is valid.

Now consider the second term on the right-hand side of (4.35). From (4.32) we have

(4.38)

$\left\|\int_{t}^{t+h} U(t+h, \tau) f(\tau, v(\tau)) d \tau\right\|_{Y_{0}} \leq \lambda_{s} \lambda_{s}^{\prime} \exp 2\left(\lambda_{N}+\mu_{N} d\right) \int_{t}^{t+h}\|f(\tau, v(\tau))\|_{Y_{0}} d \tau$

for $0 \leq t \leq t+h<d$. We have

$$
\|f(t, v(t))\|_{Y_{0}}=\left\|h(t, \cdot, u(t, \cdot))-u(t, \cdot)-c(t) u_{t}(t, \cdot)\right\|_{2} .
$$

Since $s \geq 3$, it follows from Lemma 1 that

$$
\|u(t, \cdot)\|_{2} \leq C_{d}, \quad\left\|u_{t}(t, \cdot)\right\|_{2} \leq C_{2}, \quad 0 \leq t<d .
$$


From the expression

$$
h(t, x, u(t, x))=h(t, x, 0)+\int_{0}^{1} h_{\xi}(t, x, \lambda u(t, x)) u(t, x) d \lambda
$$

we have

$$
\|h(t, \cdot, u(t, \cdot))\|_{2} \leq\|h(t, \cdot, 0)\|_{2}+\int_{0}^{1}\left\|h_{\xi}(t, \cdot, \lambda u(t, \cdot))\right\|_{2, u l}\|u(t, \cdot)\|_{2} d \lambda .
$$

From inequalities (3.8), (4.39) we obtain a constant $C_{2}>0$ such that

$$
\left\|h_{\xi}(t, \cdot \lambda u(t, \cdot))\right\|_{2, u l} \leq C_{2}, \quad 0 \leq t<d, 0 \leq \lambda \leq 1
$$

Therefore

$$
\|h(t, \cdot, u(t, \cdot))\|_{2} \leq\|h(t, \cdot, 0)\|_{2}+C_{3}, \quad 0 \leq t<d,
$$

and so there is a constant $C_{4}>0$ such that $\|f(t, v(t))\|_{Y_{0}} \leq C_{4}$ for all $0 \leq t<d$. Thus by (4.38) we obtain

$$
\lim _{t, t+h \rightarrow d}\left\|\int_{t}^{t+h} U(t+h, \tau) f(\tau, v(\tau)) d \tau\right\|_{Y_{0}}=0 .
$$

Now consider the last term on the right-hand side of (4.35). There is a constant $C_{5}>0$ such that

$$
\|[U(t+h, \tau)-U(t, \tau)] f(\tau, v(\tau))\|_{Y_{0}} \leq C_{5}\|[U(t+h, \tau)-U(t, \tau)] S f(\tau, v(\tau))\|_{z} .
$$

By $[8$, Lemma 2.5] the function $\tau \rightarrow f(\tau, v(\tau))$ is strongly measurable from $[0, d)$ to $Y_{0}$. So if we let $z(\tau)=S f(\tau, v(\tau))$, then $z(\tau)$ is strongly measurable from $[0, d)$ to $Z$. Now define

$$
\psi(t, \eta, \tau)=\|[U(t, \tau)-U(\eta, \tau)] z(\tau)\|_{z}, \quad 0 \leq \tau \leq \eta \leq t<d .
$$

Then for each $(t, \eta)$ the function $\psi(t, \eta, \tau)$ is measurable in $\tau$ and for each $\tau$ it is continuous in $(t, \eta)$. Thus for each $\tau$ we have (see (4.36))

$$
\lim _{t, \eta \rightarrow d^{-}} \psi(t, \eta, \tau)=0
$$

and by Lebesgue's Dominated Convergence Theorem

$$
\lim _{t, \eta \rightarrow d^{-}} \int_{0}^{\eta} \psi(t, \eta, \tau) d \tau=0
$$

It follows that

$$
\lim _{t, t+h \rightarrow d^{-}} \int_{0}^{t}\|[U(t+h, \tau)-U(t, \tau)] f(\tau, v(\tau))\|_{Y_{0}} d \tau=0 .
$$

Thus from (4.35) we obtain

$$
\lim _{t, t+h \rightarrow d^{-}}\|v(t+h)-v(t)\|_{Y_{0}}=0
$$

and this proves (4.33). Using (4.26) and (4.33) we obtain (4.34). Q.E.D.

REMARK 3. Theorem 4 is also valid for $s=2$ if we assume, in addition to $\left(\mathrm{c}^{*}\right)$, that $c \in C^{1}[0, \infty)$. In this case we replace $\Lambda(t)$ and $f(t, w)$ in (4.26) by

$$
\tilde{\Lambda}(t)=\left(\begin{array}{cc}
0 & -I \\
-M_{0}(t) D_{x}^{2} & c(t)
\end{array}\right), \quad D(\tilde{\Lambda}(t))=H^{2} \times H^{1},
$$


and

$$
\tilde{f}(t, w)(x)=\left(\begin{array}{c}
0 \\
h\left(t, x, w^{0}(x)\right)-w^{0}(x)
\end{array}\right), \quad w=\left(w^{0}, w^{\prime}\right) \in Y_{0} .
$$

REMARK 4. When the left-hand side of (1.1) does not explicitly depend on $u(t, x)$, then we can considerably simplify the expressions (4.2), (4.4) which occur in the growth assumption $\left(\mathrm{c}^{*}\right)$ on $c(t)$. We illustrate this in the following theorem, which concerns the equation

$$
\begin{gathered}
u_{t t}(t, x)+c(t) u_{t}(t, x)-M\left(\int_{-\infty}^{+\infty}\left|u_{x}(t, s)\right|^{2} d s\right) u_{x x}(t, x)+u(t, x) \\
=h(t, x), \quad 0<t<\infty, x \in \mathbf{R} .
\end{gathered}
$$

THEOREM 5. Let $s \geq 3$ (see Remark 3 for the case $s=2$ ). Assume that $(\mathrm{M})$ is satisfied and let $0<\varepsilon_{0}<+\infty$ be given. Suppose $h \in C\left([0, \infty) ; H^{2}\right)$ and define for $t \geq 0$ the quantities

$$
\begin{aligned}
c_{0} & =\varepsilon_{0} m_{0}^{-2} \sup \left\{\left|M^{\prime}(\lambda)\right|: 0 \leq \lambda \leq \varepsilon_{0} m_{0}^{-1}\right\}, \\
R_{0}(t) & =\frac{1}{\sqrt{m_{0}}}\left\{\varepsilon_{0}+\frac{4}{c_{0}} \int_{0}^{t}\|h(\tau)\|_{0}^{2} d \tau\right\}^{1 / 2}, \\
K_{0}(t) & =\frac{1}{m_{0}}\left\{\varepsilon_{0}+\frac{2}{c_{0}} \int_{0}^{t}\left\|h_{x x}(\tau)\right\|_{0}^{2} R_{0}(\tau) d \tau\right\} \\
L_{0}(t) & =\sup \left\{\left|M^{\prime}(\lambda)\right|: 0 \leq \lambda \leq R_{0}^{2}(t)\right\} .
\end{aligned}
$$

Suppose that $c(t)$ satisfies

$$
c \in C[0,+\infty) \quad \text { and } \quad c(t)>m_{0}^{-1} L_{0}(t) R_{0}(t) \sqrt{K_{0}(t)}, \quad t \geq 0 .
$$

For each $\varphi \in H^{2}, \psi \in H^{1}$ define

$$
\Phi(\varphi, \psi)=\|\varphi\|_{0}^{2}+\left\|D^{2} \varphi\right\|_{0}^{2}+\|\psi\|_{1}^{2}+M^{*}\left(\|D \varphi\|_{0}^{2}\right) .
$$

Then for each $\left(u_{0}, u_{1}\right) \in H^{s+1} \times H^{s}$ satisfying $\Phi\left(u_{0}, u_{1}\right) \leq \varepsilon_{0}$, there is a unique global classical solution $u(t, x)$ of $(4.40),(1.2)$ on $[0, \infty) \times \mathbf{R}$ such that $(4.25)$ holds.

Since the proof is entirely similar to Lemma 1 and Theorem 4 , we omit the details.

5. Boundedness of solutions. In Theorems 4 and 5 we see that if the initial data is "small", in the sense that $\Gamma\left(u_{0}, u_{1}\right)$ or $\Phi\left(u_{0}, u_{1}\right)$ is small, then we obtain global solutions provided $c(t)$ grows rapidly enough. However, it is not inherent in these arguments that the solution must remain small. Indeed, the forcing term is allowed to grow with respect to $t$. So in order to establish boundedness of the solutions we need a separate argument.

THEOREM 6. Suppose that the hypotheses of Theorem 4 are satisfied. Suppose also that in assumption $\left(\mathrm{h}^{*}\right)$ we have

$$
a_{1} \in L^{2}[0, \infty) \quad \text { and } \quad h_{0} \in L^{2}\left([0, \infty) ; H^{0}\right) .
$$

Then for each $\left(u_{0}, u_{1}\right) \in H^{s+1} \times H^{s}$ satisfying $\Gamma\left(u_{0}, u_{1}\right) \leq \varepsilon_{0}$, the corresponding solution $u(t, x)$ of $(1.1),(1.2)$ is bounded on $[0, \infty) \times \mathbf{R}$. 
Proof. Define

$$
R(\infty)=\frac{1}{\sqrt{m_{0}}}\left\{\varepsilon_{0}+\frac{4}{c_{0}} \int_{0}^{\infty}\left\|h_{0}(\tau)\right\|_{0}^{2} d \tau\right\}^{1 / 2} \exp \left[\frac{2}{c_{0}} \int_{0}^{\infty} a_{1}^{2}(\tau) d \tau\right]
$$

Then by (4.2), (4.18) we have

$$
|u(t, x)| \leq \sqrt{2} R(t) \leq \sqrt{2} R(\infty), \quad 0 \leq t<\infty, x \in \mathbf{R} . \quad \text { Q.E.D. }
$$

REMARK 5. A similar result on boundedness holds for solutions of (4.40), (1.2). In addition to the hypotheses of Theorem 5 , let us assume that $h \in L^{2}\left([0, \infty) ; H^{0}\right) \cap$ $L^{1}\left([0, \infty) ; H^{0}\right)$ and $h_{x x} \in L^{1}\left([0, \infty) ; H^{0}\right)$. Then for each $\left(u_{0}, u_{1}\right) \in H^{s+1} \times H^{s}$ satisfying $\Phi\left(u_{0}, u_{1}\right) \leq \varepsilon_{0}$ the corresponding solution $u(t, x)$ of $(4.40),(1.2)$ and also $u_{t}(t, x)$ are bounded on $[0, \infty) \times \mathbf{R}$.

NOTE ADDED IN PROOF. Since the submission of this manuscript, three other important publications on this subject have been brought to my attention. Two works by T. Nishida, A note on the nonlinear vibration of an elastic string, Mem. Fac. Engrg. Kyoto Univ. 34 (1971) and Nonlinear vibration of an elastic string. II (preprint), concern the global existence of analytic solutions of the undamped initial-boundary value problem corresponding to (1.1) on a bounded interval $0 \leq x \leq L$. J. M. Greenberg and S. C. Hu, The initial-value problem for $a$ stretched string, Quart. Appl. Math. 38 (1980), 289-311, consider the undamped pure initial-value problems $(1.1),(1.2)$ with $h(x, t, u)=u$. For special types of nonlinearities $M(\lambda)$ and certain classes of initial conditions (1.2), they obtain global existence and asymptotic behavior of solutions.

\section{REFERENCES}

1. R. A. Adams, Sobolev spaces, Academic Press, New York, 1975.

2. J. Ball, Initial-boundary value problems for an extensible beam, J. Math. Anal. Appl. 42 (1973), 61-90.

3. __ Stability theory for an extensible beam, J. Differential Equations 14 (1973), 399-418.

4. R. W. Dickey, Infinite systems of nonlinear oscillation equations related to the string, Proc. Amer. Math. Soc. 23 (1969), 459-468.

5. __, Infinite systems of nonlinear oscillation equations with linear damping, SIAM J. Appl. Math. 19 (1970), 208-214.

6. __ Dymamic stability of equilibrium states of an extensible beam, Proc. Amer. Math. Soc. 41 (1973), 94-102.

7. W. E. Fitzgibbon, Global existence and boundedness of solutions to the extensible beam equations, SIAM J. Math. Anal. 13 (1982), 739-745.

8. T. R. J. Hughes, Tosio Kato and Jerrold Marsden, Well-posed quasi-linear second onder hyperbolic systems with applications to non-linear elastodynamics and general relativity, Arch. Rational Mech. Anal. 63 (1977), 273-294.

9. T. Kato, Linear evolution equations of "hyperbolic type", J. Fac. Sci. Univ. Tokyo Sect. IA Math. 17 (1970), 241-258.

10. __ Linear evolution equations of hyperbolic type. II, J. Math. Soc. Japan 25 (1973), 648-666.

11. _ The Cauchy problem for quasi-linear symmetric hyperbolic systems, Arch. Rational Mech. Anal. 58 (1975), 181-205.

12. J. L. Lions, On some questions in boundary value problems of mathematical physics, in Contemporary Developments in Continuum Mechanics and Partial Differential Equations (G. M. de LaPenha and L. A. J. Mederios, eds.), North-Holland Math. Stud., vol. 30, North-Holland, Amsterdam, 1978, pp. 284-346.

13. , Quelques methodes de resolution des problèmes aux limites non linéaires, Dunod, Paris, 1969.

14. R. Narasimha, Non-linear vibration of an elastic string, J. Sound Vibration 8 (1968), 134-146. 
15. D. W. Oplinger, Frequency response of a nonlinear stretched string, J. Acoust. Soc. Amer. 32 (1960), 1529-1538.

16. S. Woinowsky-Krieger, The effect of an axial force on the vibration of hinged bars, J. Appl. Mech. 17 (1950), 35-36.

DePARTMENT OF MAThematics, Statistics AND COMPUTER SCIENCE, UNiversity OF Illinois, ChicAgo, ILlinois 60680 